\title{
Numerical Study on Oil Flow in the Vicinity of Discharge Reed Valve of Air Compressor
}

\author{
Susumu Degawa ${ }^{1}$, Koichi Nishibe ${ }^{2}$, Suguru Shiratori ${ }^{2}$, Itsuhei Kohri ${ }^{2},{\text { Akemi } \text { Ito }^{2} \text { and Akinori Nishimura }}^{3}$ \\ ${ }^{1}$ Graduate School of Integrative Science and Engineering ,Tokyo City University \\ 1-28-1 Tamazutsumi Setagaya-ku, Tokyo 158-8557, Japan \\ g1881041@tcu.ac.jp \\ ${ }^{2}$ Department of Mechanical Engineering, Tokyo City University \\ 1-28-1 Tamazutsumi Setagaya-ku, Tokyo 158-8557, Japan \\ Knishibe@tcu.ac.jp; sshraor@tcu.ac.jp; ikohri@tcu.ac.jp; aito@tcu.ac.jp \\ ${ }^{3}$ Nabtesco Automotive Corporation \\ 2931-18, Urago-cho 5-chome, Yokosuka, Kanagawa 237-0062, Japan \\ Akinori_Nishimura@nabtesco-automotive.com
}

\section{Extended Abstract}

Air brakes are used in the braking systems of heavy-duty vehicles because a higher braking force can be achieved with the air braking system than a hydraulic system. Recently, all reciprocating device including that of the air braking system is required further improvement of efficiency to save energy. Low-tension piston rings are known as one of the solutions. However, the solution induces increasing oil consumption and performance degradation of air dryers installed downstream of the air braking system cause problems. A part of the lubricating oil applied to sliding surfaces is atomized, enters the compression chamber, and is released downstream with compressed air from the discharge reed valve during operation of the reciprocating air compressor, which is the actuator of the air braking system. Therefore, reduction of amount of oil particles passing though the discharge valve is one of the critical issues. To date, several studies on the flow patterns of oil flow and the behavior of oil particles in air conditioner or refrigeration system have been conducted. Zimmermann and Hrnjak conducted an experiment to visualize the break up pattern of oil film in the vicinity of discharge reed valve during operating scroll compressor [1]. Xu and Hrnjak investigated the behavior of oil particles inside a scroll compressor discharge plenum by conducting both computational fluid dynamics (CFD) and visualization [2]. However, these studies have confirmed the behaviour of oil particles downstream of the discharge reed valve only in fixed degree of valve opening. Moreover, detailed investigations of the problem and its solutions are inadequate and unclear.

Therefore, the present study attempts to investigate methods to reduce oil consumption by demonstrating the flow field and behaviour of oil particles in the compression chamber and vicinity of the discharge reed valve using unsteady multiphase flow analysis with discrete phase models in CFD. The flow field was assumed as a two-dimensional compressible turbulence flow. The time variation of the compression chamber volume and degree of discharge valve opening were simulated by applying the moving mesh method as boundary condition in accordance with the test reciprocating compressor in the experiment. After validating the CFD simulations by comparisons with experimentally obtained pressure fluctuations around the discharge reed valve, the influence of the valve motion, compressor speed, and oil particle diameter on the flow field and trajectory of the oil particles was analysed. Furthermore, the percentage of oil particles passing through the discharge reed valve was also investigated. The number of oil particles passing through the discharge reed valve depends on the size of oil particle diameter and compressor speed.

\section{References}

[1] A. J. P. Zimmermann and P. S. Hrnjak, "Source Identification and In Situ Quantification of Oil-Refrigerant Mist Generation by Discharge Valve Opening Process" in International Compressor Engineering Conference, Purdue, 2014, Paper 2367.

[2] Jiu Xu and P. S. Hrnjak, "Visualization and Simulation of Oil Flow in a Scroll Compressor Plenum," in International Compressor Engineering Conference, Purdue, 2016, Paper 2507. 